\title{
Má Alimentação e Obesidade nos Alunos dos Cursos de Saúde de Uma Instituição Privada de Vitória da Conquista
}

\author{
Halisson Naninne Morais Salomão ${ }^{1}$; Felipe Oliveira Bittencourt ${ }^{2}$
}

\begin{abstract}
Resumo: O presente projeto tem por objetivo geral identificar o perfil da obesidade em acadêmicos de cursos de saúde de uma instituição privada de Vitória da Conquista/BA. E como objetivo específico: identificar o perfil da má alimentação e obesidade em estudantes universitários de 18 a 25 anos, como sendo as pessoas mais interessadas e entendidas do assunto. Trata-se de uma pesquisa do tipo analítica com delineamento transversal e abordagem quantitativa, tendo uma amostra de 100 indivíduos, de cursos de saúde variados. Trabalho baseado dentro do projeto de extensão, sobre "O perfil epidemiológico da obesidade em Vitória da Conquista/BA", aprovado pelo Comitê de Ética em Pesquisa da FAINOR e autorizada pelo CEP. Os dados foram coletados em formulário próprio de questionário de hábitos alimentares. Para a descrição das variáveis contínuas foi utilizada a média aritmética, com seu respectivo desvio padrão e, para variáveis categóricas, número absoluto e frequência relativa. Como resultado desse trabalho evidenciou-se a prevalência de uma alimentação inadequada entre os participantes da amostra $(65,5 \%)$, um grande indicie global de sobrepeso $(39,6 \%)$ e obesidade $(13,6 \%)$, estando bem acima dos valores encontrados na maioria das literaturas, além de uma prevalência de sobrepeso na população masculina da amostra (40,6\%). Considera-se assim que os resultados desse estudo indicam a necessidade de intervenção no ambiente universitário, visto que a grande prevalência de problemas relacionados ao peso e a má alimentação tendem a proporcionar o desenvolvimento de outras patologias, influenciando na queda da qualidade de vida dos estudantes universitários, e consequentemente em seu rendimento acadêmico.
\end{abstract}

Palavras-chave: Obesidade. Má Alimentação. Ensino Superior.

\section{Bad Eating Habits and Obesity in Students of Health Courses of a Private Institution in Vitória da Conquista}

\begin{abstract}
The present project aims to identify the profile of obesity among academics of health courses at a private institution in Vitória da Conquista / BA. And as a specific objective: to identify the profile of poor diet and obesity in university students aged 18 to 25 , as being the most interested and knowledgeable people on the subject. This is an analytical type research with a cross-sectional design and a quantitative approach, with a sample of 50 individuals from varied health courses. Work based on the extension project, on "The epidemiological profile of obesity in Vitória da Conquista / BA", approved by the Research Ethics Committee of FAINOR and authorized by CEP. Data were collected in the form of a questionnaire of eating habits. For the description of the continuous variables, the arithmetic mean was used, with its respective standard deviation and, for categorical variables, absolute number and relative frequency. As a result of this study, the prevalence of an inadequate diet among the participants of the sample $(65.5 \%)$ was evidenced, a large overall index of overweight $(39.6 \%)$ and obesity $(13.6 \%)$, being well above the Values found in most of the literature, as well as a prevalence of overweight in the male sample (40.6\%). Thus, the results of this study indicate the need for intervention in the university environment, since the high prevalence of problems related to weight and poor nutrition tend to provide the development of other pathologies, influencing the drop in quality of life of students, and consequently in their academic performance.
\end{abstract}

Keywords: Obesity. Bad Eatings Habilits Higher Education.

\footnotetext{
1 Graduando o curso de Enfermagem da FAINOR - Faculdade Independente do Nordeste, Vitória da Conquista, Bahia. halissonnaninne@gmail.com.

${ }^{2}$ Bacharel em Farmácia, Especialista em Farmácia Hospitalar, Especialista em Farmácia Magistral e Mestre em Saúde e Ambiente. felipe@fainor.com.br
} 
Id on Line Revista Multidisciplinar e de Psicologia

Id on Line Multidisciplinary and Psycology Journal

\section{Introdução}

A obesidade está entre os maiores problemas de saúde pública, uma vez que se enquadra e está fortemente relacionada com doenças crônicas como: HAS, Diabete Melitus, problemas cardiovasculares e outros. A obesidade é caracterizada pelo aumento excessivo da gordura corporal causado pelo consumo demasiado de alimentos com alto teor de gordura e o não gasto energético (PINHO et. al, 2013). Com sua incidência crescente, torna-se alarmante o problema, visto que deve-se levar em consideração sua evolução e associações múltiplas que a acompanham, visto que sua etiologia é multifatorial, envolve diversos aspectos biológicos, históricos, ecológicos, políticos, socioeconômicos, psicossociais e culturais, além dos fatores principais já expostos. A obesidade aumenta o risco de mortalidade relacionada a problemas cardiovasculares e reduzem a qualidade de vida (ARQ BRAS CARDIOL, 2009).

Evidências atuais mostram que esse problema afeta milhões de brasileiros e o alto número de indivíduos com obesidade abdominal mostra que a proporção do problema vem afetando parâmetros epidemiológicos alarmantes (ROMANZINI et. al, 2011). Essa patologia é considerada, atualmente, a maior desordem nutricional tanto em países desenvolvidos, quanto nos que estão em desenvolvimento, devido a sua incidência, provocando assim impactos negativos nos Sistemas Públicos de Saúde. Segundo predições recentes, o impacto da obesidade, suas consequências e seus possíveis desenvolvimentos tecnológicos, podem levar a atual geração da população jovem a ter uma expectativa de vida menor do que as anteriormente registradas (ARQ BRAS CARDIOL, 2009).

A saúde pessoal e a obesidade são condições relacionadas à má alimentação. As pessoas têm a opção de escolher o " que" comer e o " quanto" comer, mas os abusos alimentares podem trazer prejuízos à saúde. A relação entre a fome e compulsão são que a fome é uma condição dada a pessoa pela pobreza, que não tem o direito de escolha, enquanto a compulsão é justamente a escolha do comportamento abusivo e excessivo no ato de se alimentar (ORTIGOZA, 2008).

Nesta perspectiva, levando em consideração os dados a serem coletados, o estudo visa a identificação do perfil epidemiológico da obesidade em alunos de uma instituição privada de ensino superior do município de Vitória da Conquista/BA e analisar a relação entre a sua 
Id on Line Revista Multidisciplinar e de Psicologia

Id on Line Multidisciplinary and Psycology Journal

alimentação com fenômenos associados à obesidade pois, teoricamente, esses são indivíduos mais esclarecidas e interessados na temática e os nos possíveis impactos na saúde.

\section{Metodologia}

Este trabalho demostra as características de determinada população ou fenômenos de abordagem qualiquantitativa, com pesquisa de campo, exploratória com abordagem descritiva.

O local de estudo será uma instituição de ensino superior privada do município de Vitória da Conquista/BA situado na região sudoeste do Estado da Bahia. Os participantes do presente estudo foram selecionados por faixa etária de ambos os sexos, de 19 a 25 anos, representando um total de 100 indivíduos. O estudo foi realizado através da aplicação de questionários validado de Ferreira, M.G. et. al. (2010) aos alunos, com a análise de dados feita primeiramente através da estatística descritiva dos dados e posteriormente a análise Kruskal Wallis para verificar uma possível associação entre as variareis do estudo (nível de significância de 0,05$)$, sendo que todas foram analisados no software estatístico SPSS 23,0 da IBML.

A análise dos dados se baseia nos depoimentos colhidos através do questionário aplicado e cálculo de Índice de Massa Corpórea - IMC analisando de forma comparativa com o que foi pesquisado e obtido pelo referencial teórico, o que busca considerar os objetivos propostos no trabalho.

A coleta foi realizada em setembro de 2016 durante o período da pesquisa. A pesquisa documental foi realizada com a mesma frequência de registro da pesquisa observacional.

Utilizando-se como técnicas de coleta de dados, questionário estruturado e a observação não participante, visa então verificar a dinâmica alimentar e o perfil da obesidade desses alunos. A coleta de dados realizada na pesquisa tomará como base às respostas dos questionários, obtendo resultados a serem discutidos e analisados. 


\section{Resultados e Discussão}

O presente estudo foi representativo dentro do universo de estudantes de uma universidade particular, em Vitória da Conquista-Bahia, podendo ser comparado com outros de universitários de regiões com características socioeconômicas semelhantes em virtude de sua validação científica.

Participaram da pesquisa 100 acadêmicos, com prevalência de $66,4 \%$ do sexo feminino. A amostra foi composta por $71,8 \%$ de estudantes que trabalham ou realizam alguma atividade remunerada. Entre os quantitativos da amostra a maioria dos participantes da pesquisa eram $49,1 \%$ eram de solteiros. Identificou-se também entre os participantes que $65,5 \%$ tinham uma alimentação inadequada ao longo do dia e que $54,5 \%$ não executavam nenhum tipo de atividade física regular (TABELA 1).

Tabela 1 - Perfil Sociodemográfico dos entrevistados.

\section{Variáveis}

Frequência

Porcentagem

\section{Sexo}

Feminino

66,4

Masculino

\section{Estado Civil}

Casado

Divorciado

Solteiro

Viúvo

\section{Que trabalham}

Sim

Não

\section{Continua....}


Id on Line Revista Multidisciplinar e de Psicologia

Id on Line Multidisciplinary and Psycology Journal

\section{Alimentação}

Adequada

Inadequada

Prática de Atividade Física

Sim

Fonte: Pesquisa.

Esse resultado aponta para uma preponderância do gênero feminino no ambiente universitário, dado compatível com o apontado em outros estudos como os de Maso e Feitosa (2013), que observaram em sua pesquisa em faculdades particulares, que a maioria dos estudantes universitários pesquisados era do sexo feminino, com $68 \%$ da amostra, e os de Fontes, Pontes e Vianna (2012), que verificaram em sua pesquisa uma amostra de 55,9\% de universitários do sexo feminino.

Confirmando essa tendência, dados do INEP, Instituto Nacional de Estudos e Pesquisas Educacionais Anísio Teixeira (2005), revelam que em treze anos a população feminina matriculada no ensino superior cresceu $22 \%$, atualmente cerca de $56 \%$ das matrículas pertencem às mulheres.

Em relação ao estado civil, os dados de diversos trabalhos indicam a mesma dinâmica encontrada nesse estudo, pois apresentam uma preponderância de solteiros entre os estudantes universitários, sendo que no estudo de Duarte, Almeida e Martins (2013) esse índice apresenta alta porcentagem $(85,2 \%)$ e em outros como Maso e Feitosa (2013) apresenta-se índice mais próximo $(49,7 \%)$, do verificado nesse estudo.

Em relação ao trabalho, o estudo de Quintino et al (2014) corrobora com os resultados encontrados nessa pesquisa, pois verifica-se que a maior parte dos estudantes participantes da amostra trabalham, sendo que $61,0 \%$ da amostra indicou realizar alguma atividade remunerada. No entanto, em discordância com esses resultados o estudo de Duarte, Almeida e Martins (2013), mostraram que 73,3\% dos estudantes de uma universidade particular em Goiânia, não trabalhavam.

Quanto a prática de atividade física, Quintino et al (2014) observou, em sua pesquisa frente a universitários, que a grande maioria da sua amostra $(81,4 \%)$ apresentam 
Id on Line Revista Multidisciplinar e de Psicologia

Id on Line Multidisciplinary and Psycology Journal

comportamento ativo, ou seja realizam atividades físicas, sendo que somente $18,6 \%$ não realizam atividade física. Santos et al (2014) também enumera em seu estudo resultado similar pois observou, em sua pesquisa frente a universitários, que a maioria da sua amostra $(50,83 \%)$ não realizavam atividade física.

Em relação a alimentação saudável, apresentando semelhança nos resultados, Henn et al (2014), aborda que em sua pesquisa frente aos acadêmicos de uma instituição de ensino superior, do total dos entrevistados, $25 \%$ acham que sua alimentação é saudável, sendo que os outros $75 \%$ acham que não é saudável ou às vezes saudável.

Sobre essa questão Ramalho, Dalamaria e Souza (2012), enumera que o consumo alimentar dos estudantes universitários muitas vezes é baseado na ingestão de fast foods, pelo fato de não terem muito tempo para se alimentar corretamente devido a sua rotina ser muito corrida.

Diante desses dados, Vieira et al (2007), aponta que com essa prática alimentar, há estudos que mostram que os jovens não praticam atividades físicas, aumentando a chance de ficarem obesos, tendo um excesso calórico em virtude do aumento do consumo, há uma diminuição do gasto, via sedentarismo.

A Tabela 2 apresenta os dados globais de IMC coletados frente aos estudantes participantes da pesquisa. Como resultado da avaliação percebe-se dentro da amostra 44,1\% $(n=49)$ dos estudantes estão com peso normal, 39,6\% $(n=44)$ apresentam sobrepeso, 13,6\% (n=15) já estão com diagnostico de obesidade, e 1.8\% (n=02) estão com baixo peso.

Tabela 2 - Resultado Global do IMC averiguado entre entrevistados.

\begin{tabular}{lcc}
\hline Variável & Frequência & Porcentagem (\%) \\
\hline Resultado de IMC & & \\
\hline Abaixo do Peso & 2 & 1,8 \\
\hline Obesidade & 15 & 13,6 \\
\hline Peso Normal & 49 & 44,1 \\
\hline Sobrepeso & 44 & 39,6 \\
\hline
\end{tabular}

Fonte: Pesquisa. 
Id on Line Revista Multidisciplinar e de Psicologia

Id on Line Multidisciplinary and Psycology Journal

Esses dados mostram uma realidade preocupante, pois observa-se que os problemas de sobrepeso e obesidade, já estão alcançando mais da metade dos estudantes que fazem parte da amostra desse trabalho.

Em comparação com resultados de outros estudos, como o de Feitosa et al (2010), onde aponta-se que $17,9 \%$ do total apresentavam sobrepeso/obesidade entre estudantes universitários, esse estudo mostra que seus dados são ainda mais alarmantes.

Essa divergência também é verificada na pesquisa de Fontes, Pontes e Vianna (2012), onde a prevalência de sobrepeso foi de 15,30\% e obesidade com 5,70\%. Bem como, no estudo de Pereira, Terra e Rodrigues (2015), que verificaram em sua amostra, 15\% de estudantes com sobrepeso e 5\% de estudantes obesos. Ambos apresentando níveis bem menores que os aqui diagnosticados.

No entanto, o trabalho de Ribeiro et al (2015), observou-se que 58,1\% da amostra apresentou excesso de peso, apresentando grande prevalência de sobrepeso $(41,2 \%)$ e de obesidade $(16,9 \%)$, tal qual foi verificado nesse estudo. Outros estudos que apresentam dados semelhantes são os de Vieira e Silva (2014), onde numa avaliação de 62 universitários constatou que 51,6\% da amostra apresentava excesso de peso, Paixão, Dias e Prado (2009) que encontraram a prevalência de sobrepeso 47,68\% em estudantes de Recife/PE, após avaliarem 253 indivíduos, Oliveira et al (2011), que em pesquisa com estudantes, detectou prevalência de excesso de peso em 51,04\%, numa amostra de 145 indivíduos.

Dessa forma, cabe destacar que a prevalência de excesso de peso encontrada no presente estudo e considerada elevada quando comparada com outros estudos já sinalizados. No entanto, os dados da Vigilância de Fatores de Risco e Proteção para Doenças Crônicas por Inquérito Telefônico (VIGITEL), revelaram que no ano de 2012, 48,4\% dos adultos com 12 ou mais anos de estudo apresentaram excesso de peso (BRASIL, 2013).

Na Tabela 3 está descrito o resultado da associação entre IMC e a variável sexo. Como resultado dessa associação, percebe-se a prevalência de sobrepeso entre os homens $(40,6 \%)$, sendo que esse índice é muito similar ao encontrado nas mulheres $(39,7 \%)$. Em relação à obesidade, entre os homens é de $27 \%$ da amostra, enquanto entre as mulheres atinge somente $6,8 \%$. Quanto aos estudantes que apresentaram peso normal, a prevalência é maior no sexo feminino $(50,7 \%)$ que no masculino $(32,4 \%)$. Estudantes que se apresentaram abaixo do peso foram somente duas mulheres $(2,8 \%)$, não sendo verificado nenhum caso entre os homens. 
Id on Line Revista Multidisciplinar e de Psicologia

Id on Line Multidisciplinary and Psycology Journal

Tabela 3 - Resultado da Associação entre as variáveis IMC e Sexo dos entrevistados.

\begin{tabular}{llcr}
\hline \hline Sexo & & Frequência & Porcentagem \\
\hline Feminino & Abaixo do peso & 2 & 2,8 \\
\cline { 2 - 4 } & Obesidade & 5 & 6,8 \\
\cline { 2 - 4 } & Peso normal & 37 & 50,7 \\
\cline { 2 - 4 } & Sobrepeso & 29 & 39,7 \\
\hline Masculino & Abaixo do peso & 00 & 0,0 \\
\cline { 2 - 4 } & Obesidade & 10 & 27,0 \\
\cline { 2 - 4 } & Peso normal & 12 & 32,4 \\
\cline { 2 - 4 } & Sobrepeso & 15 & 40,6 \\
\hline
\end{tabular}

Fonte: Pesquisa.

Segundo Gigante et al (2006), no Brasil, evidências revelam que as prevalências de excesso de peso e obesidade tendem a aumentar na população adulta, pelo fato de ter uma queda significativa no metabolismo, de forma diferenciada, entre os sexos. Assim, no período entre 1974-75, a obesidade entre os homens era maior em relação as mulheres. Enquanto que a população feminina teve prevalência mais elevada no início do período de 2002 em 50\%. (GIGANTE et. al., 2006).

O estudo de Oliveira et. al. (2011), corroborando com os resultados desse trabalho, apontam em sua pesquisa que o fator gênero estabeleceu um perfil específico, sendo os homens com $57,3 \%$ de prevalência apresentaram excesso de peso, e as mulheres, na maioria, foram classificadas como normal $(64,3 \%)$.

Ribeiro et. al. (2015) também aponta resultado semelhantes em sua pesquisa, indicando a prevalência de sobrepeso $(48,8 \%)$ e obesidade $(22,6 \%)$ para os homens e de peso normal $(61,5 \%)$ e sobrepeso $(28,9 \%)$ para as mulheres.

Nessa mesma ótica Vieira e Silva (2014), ao realizarem pesquisa com 62 estudantes universitários averiguaram frente a sua amostra que no sexo masculino a maior parte dos pesquisados apresentavam excesso de peso $(66,7 \%)$, enquanto entre as mulheres a aferição indicava peso normal em mais da metade das pesquisadas $(56,1 \%)$. 
Mesmo com menor prevalência que o observado nesta pesquisa, os estudos realizados na Líbia (YAHIA, et al., 2008) e na cidade de Santa Catarina, Brasil (LOOCK, et. al., 2006) confirmam a maior prevalência de sobrepeso entre universitários do sexo masculino, quando comparado com o sexo feminino. Eisenmann, et. al., (2004) observaram também resultado semelhante em relação a excesso de peso e sexo entre estudantes americanos. Consideramos que os padrões culturais de valorização da estética feminina podem proteger as mulheres do sobrepeso nessa fase de vida.

No entanto, quando comparados com dados nacionais (BRASIL, 2013), que revelaram que $47,3 \%$ e $39,5 \%$ de homens e mulheres, respectivamente, apresentaram excesso de peso, observa-se que esses resultados diferem dos encontrados nesse estudo.

Esses valores também diferem dos resultados encontrados no estudo de VelasquezMelendez, Pimenta e Kag (2004), realizado em Belo Horizonte, em que a prevalência de sobrepeso foi observada em $38,7 \%$ da população, e a obesidade em $10,2 \%$, sendo que no sexo masculino os valores de excesso de peso apresentaram-se menores $(36,8 \%)$, e entre o sexo feminino foi encontrado $40,6 \%$ de sobrepeso/obesidade.

A Tabela 4 mostra os resultados da análise bivariada para testar associações entre IMC e a práticas alimentares diária dos estudantes universitários.

Tabela 4 - Resultado da Associação entre as variáveis IMC e Alimentação dos entrevistados.

\begin{tabular}{llcr}
\hline Alimentação & \multicolumn{2}{c}{ Frequência } & Porcentagem \\
\hline Adequada & Abaixo do peso & 02 & 5,3 \\
\cline { 2 - 4 } & Obesidade & 05 & 13,1 \\
\cline { 2 - 4 } & Peso normal & 19 & 50,0 \\
\cline { 2 - 4 } & Sobrepeso & 12 & 31,6 \\
\hline Inadequada & Abaixo do peso & 00 & 0,0 \\
\cline { 2 - 4 } & Obesidade & 10 & 13,9 \\
\cline { 2 - 4 } & Peso normal & 30 & 41,6 \\
\cline { 2 - 4 } & Sobrepeso & 32 & 44,5 \\
& & & \\
& & &
\end{tabular}

Fonte: Pesquisa Própria. 
Id on Line Revista Multidisciplinar e de Psicologia

Id on Line Multidisciplinary and Psycology Journal

Dentro dessa análise percebe-se que a prevalência de sobrepeso $(44,5 \%)$ e de obesidade $(13,9 \%)$ está no grupo de estudantes que com alimentação inadequada. Em relação ao peso normal, ocorre maior frequência desse grupo entre os alunos que tem uma alimentação mais adequada $(50 \%)$.

Diante desses resultados, pode-se enumerar que a prática alimentar desenvolvida pelos estudantes universitários têm relação direta com o desenvolvimento de demandas relacionadas ao peso, principalmente a obesidade e o sobrepeso.

Sobre essa questão Feitosa et. al. (2010), aponta que os hábitos alimentares dos universitários são fortemente influenciados por fatores como o ingresso na universidade, pois para alguns a vida universitária implica em deixar a casa dos pais e passar a viver em moradias estudantis, devido à localização da instituição, além disso a falta de tempo para realizar refeições completas por causa das atividades acadêmicas que influenciam na escolha dos alimentos, na substituição de refeições completas por lanches práticos e rápidos, com alto valor calórico; e o estabelecimento de novos comportamentos e relações sociais.

Assim, confirmando os dados das pesquisas nacionais (BRASIL, 2014), estudos mostram que o alto consumo de alimentos ricos em gorduras e açúcares, a não adoção de hábitos alimentares saudáveis, acompanhado dosedentarismo, favorecem o aparecimento da obesidade.

Corroborando com os resultados desse trabalho, um estudo com 54 universitários na Tunísia revelou que a alimentação desses estudantes era inadequada (60\%), rica em gordura saturada, colesterol e açúcares simples, sendo que $20 \%$ dos participantes do estudo não tem horário regular para comer, 37\% apresentaram sobrepeso, e 9\% obesidade (BURRIEL, et. al., 2014 apud LEIBOVICH, 2015).

Dessa forma, em consonância com o que tem sido relatado na literatura, o presente estudo mostra uma nítida associação entre práticas alimentares menos saudáveis e obesidade existente dentro da amostra dessa pesquisa.

Portanto, mesmo diante de estudos que apontem resultados diferentes aos aqui encontrados, esse trabalho pode confirmar boa parte das tendências descritas na maior parte da literatura, apresentando a preponderância do gênero masculino em relação ao gênero feminino no desenvolvimento da obesidade e da má alimentação dentro do ambiente universitário. 
Id on Line Revista Multidisciplinar e de Psicologia

Id on Line Multidisciplinary and Psycology Journal

\section{Considerações Finais}

Diante dos conceitos, resultados e discussões realizados nesse estudo, esse trabalho buscou traçar o perfil de estudantes universitários, de modo a observar a influência dessas características na má alimentação e no desenvolvimento da obesidade, dentro de uma instituição de ensino superior.

Em relação ao perfil sociodemográfico desse estudo, analisando variáveis como sexo, estado civil, trabalho, pratica de atividades físicas e alimentação, a prevalência encontrada foi de uma amostra composta por estudantes em uma maioria de mulheres, solteiros, que trabalhavam, não praticavam atividades físicas e se tinham uma alimentação inadequada.

Quanto ao diagnostico da obesidade através do IMC, esse trabalho apresentou um alto índice de sobrepeso e de obesidade dentro da amostra, índice muito maior que o verificado na maioria das literaturas, recomendando-se assim a adoção de ações de controle de peso e maiores incentivos para a prática de um estilo de vida saudável. Em relação aos dados por sexo, os homens apresentaram maior prevalência de sobrepeso, enquanto as mulheres em sua maioria apresentaram peso normal.

Quanto a questão da influência da má alimentação frente ao desenvolvimento da obesidade, esse estudo apontou que a maior parte dos casos verificados de obesidade estão entre os estudantes que declararam se alimentar de forma inadequada. Assim, pode-se considerar que a má alimentação tem relação direta com o desenvolvimento de problemas como a obesidade e o sobrepeso.

Por fim, os resultados desse estudo indicam a necessidade de intervenção no ambiente universitário, visto que a grande prevalência de problemas relacionados ao peso e a má alimentação tendem a proporcionar o desenvolvimento de outras patologias, influenciando na piora da qualidade de vida dos estudantes universitários, e consequentemente em seu rendimento acadêmico. 
Id on Line Revista Multidisciplinar e de Psicologia

Id on Line Multidisciplinary and Psycology Journal

\section{Referências}

BRASIL. Ministério da Educação. Instituto Nacional de Pesquisas Educacionais Anísio Teixeira: ME, 2005. Disponível em:<http://www.edudatabrasil.inep.gov.br $>$ Acesso em 23/10/2016.

Ministério da Saúde do Brasil. Vigitel - Vigilância de fatores de risco e proteção para doenças crônicas por inquérito telefônico 2012. Brasília, 2013.

Ministério da Saúde. Guia alimentar para a população brasileira. Brasília: 2014.

DUARTE, F. M.; ALMEIDA, S. D. S.; MARTINS, K. A. Alimentação fora do domicílio de universitários de alguns cursos da área da saúde de uma instituição privada. Revista O Mundo da Saúde, São Paulo, n. 37(3), 2013.

FEITOSA, E. P. S.; et al. Hábitos alimentares de estudantes de uma universidade pública no nordeste, Brasil. Araraquara, 2010.

FERREIRA, M.G. et. al. Desenvolvimento de questionário de frequência alimentar para adultos, Brasil, Rev. Bras. Epidemiologia, 13(3), pag - 413-424, 2010.

FONTES, A. C. D.; PONTES, L. M.; VIANNA, R. P. T. Prevalência e Fatores associados ao Sobrepeso e Obesidade em Estudantes Universitários, Paraíba, Brasil. Coleção Pesquisa em Educação Física, vol.11, n.2, 2012.

GIGANTE, D. P.; et al. Obesidade da população adulta de Pelotas, Rio Grande do Sul, Brasil e associação com nível sócio-econômico.. Cad. Saúde Pública, Rio de Janeiro, 22(9), set, 2006.

HENN, C.; et al. Padrão Alimentar dos Acadêmicos da Faculdade de Itapiranga. I Simpósio de Agronomia e Tecnologia de Alimentos, Itapiranga, 2014.

LEIBOVICH, H. Y. Avaliação do consumo alimentar de estudantes frequentadores do Restaurante Universitário da Universidade de Brasília. Dissertação (Mestrado). Brasília, DF: Universidade de Brasília- Faculdade de Ciências da Saúde, 2015.

LOOCK, M. R; KONRAD, L. M.; SANTOS, P. D; NAHAS, M. V. Perfil da aptidão física relacionada à saúde de universitários da educação física curricular. Revista Brasileira de Cineantropometria. Desempenho Humano, v.8, n.1, p. 64-71, 2006.

MASO, M. D.; FEITOSA, F. B. Um estudo comparativo entre dados sociodemográficos e neuroticismo. Estudos e Pesquisas em Psicologia, vol.. 13, n. 3, 2013.

OLIVEIRA, R. A. R. et al. Prevalência de sobrepeso e obesidade em professores da Universidade Federal de Viçosa. Revista Fisioterapia em Movimento, out/dez, n. 24(4), 2011. 
Id on Line Revista Multidisciplinar e de Psicologia

Id on Line Multidisciplinary and Psycology Journal

PAIXÃO, Leticia A.; DIAS, Raphael M. R.; PRADO, Wagner L. Estilo de vida e estado nutricional de universitários ingressantes em cursos da área de saúde do recife/PE. Revista Brasileira de Atividade Física e Saúde, v. 15, n. 3, p. 145-150, 2009.

PEREIRA, M. A. V.; TERRA, G. D. . V.; RODRIGUES, C. A. C. Mudanças de Hábitos: Uma Análise da Composição Corporal e Nutricional em Universitários. Revista Brasileira de Obesidade, Nutrição e Emagrecimento, São Paulo. v.9. n.54. p.251-257. Nov./Dez. 2015.

PINHO, C. P. S. et al. Prevalência e fatores associados à obesidade abdominal em indivíduos na faixa etária de 25 a 59 anos do Estado de Pernambuco, Brasil. Cad. Saúde Pública, Rio de Janeiro, v. 29, n. 2, p. 313-324, fev. 2013.

QUINTINO, P. L.; et al. Estágios de mudança de comportamento para atividade física em universitários e fatores sociodemográficos associados. Revista Brasileira de Educação Física e Esporte, São Paulo, Abr-Jun, n. 28(2), 2014.

RAMAlHO, A. A.; DALAMARIA, T.; SOUZA, O. F. Consumo regular de frutas e hortaliças por estudantes universitários em Rio Branco, Acre, Brasil: prevalência e fatores associados. Rio de Janeiro, Jul 2012. Disponível em <311X2012000700018\&lng=pt\&nrm =isso\&tlng=> Acesso em 23/10/2016.

RIBEIRO, R.; et al. Prevalência de Excesso de Peso em Universitários de uma Faculdade Particular do Município de Praia Grande-SP. Revista Eletrônica Acadêmica Interinstitucional, Ano VIII - No XVIII- JUL/ 2015.

ROMANZINI, M.; PELEGRINI, A.; PETROSKI, E. L. Prevalência e fatores associados à obesidade abdominal em adolescentes. Revista Paulista de Pediatria, São Paulo, v. 29, n. 4, p. 546-552, 2011.

SANTOS, A. K. G.V.; et al. Qualidade de Vida e Alimentação de estudantes universitários que moram na região central de São Paulo sem a presença dos pais ou responsáveis. Rev. SimbioLogias, v.7, n. 10, Dez/2014.

VELÁSQUEZ-MELÉNDEZ, G.; PIMENTA, A. M.; KAG, G. Epidemiologia do sobrepeso e da obesidade e seus fatores determinantes em Belo Horizonte (MG), Brasil: estudo transversal de base populacional. Rev Panam Salud Publica, n. 16(5), 2004.

VIEIRA, A. M.; et al. Fast Food: Modificação dos hábitos alimentares. Publicado em 2007. Disponível em: <http://www.assesc.edu.br/download/4-jornada-academica/Fast_food. pdf>. Acesso em 26/10/2016.

VIEIRA, E. M. S.; SILVA, A. R. V. Excesso de Peso, Obesidade Abdominal e Níveis Pressóricos em Universitários. I Congresso Nacional de Ciências da Saúde: Avanços, Interfaces e Práticas Interrogativas, Cajazeiras-PB, 2014. 
YAHIA, N.; ACHKAR, A.; ABDALLAH, A.; RIZK, S. Eating habits and obesity among Lebanese universits students. Nutrition Journal. v.7, n.32, p.1-6, 2008.

\section{Como citar este artigo (Formato ABNT):}

SALOMÃO, H.N.M.; BITTENCOURT, F.O. Má Alimentação e Obesidade nos Alunos dos Cursos de Saúde se Uma Instituição Privada de Vitória da Conquista. Id on Line Revista Multidisciplinar e de Psicologia, Janeiro de 2017, vol.10, n.33, p.18-31. ISSN: 1981-1179.

Recebido: 23/11/2016

Aceito: $24 / 11 / 2016$ 